\title{
Utilização do método TDR-tensiômetro na obtenção da curva de retenção de água no solo
}

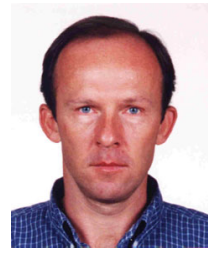

Sérgio R. L. Otto ${ }^{1}$ \& Miguel Alcaide ${ }^{2}$

1 UEPG, Pç. Santos Andrade s/n, CEP 84010-790, Ponta Grossa, PR. Fone: (42) 220-3090. E-mail: srotto@convoy.com.br (Foto)

2 Univ. de Córdoba, Apdo de Correos 3048, 14080 Córdoba, España. Fone: (957) 218513. E-mail: ag1algam@uco.es

Protocolo 119 - 19/10/1999

\begin{abstract}
Resumo: O presente trabalho teve como objetivo testar a utilização conjunta do TDR (Reflectometria no Domínio do Tempo) e do tensiômetro, na determinação da curva de retenção de água no solo. Foram determinadas várias curvas de retenção de água de um solo franco-arenoso, em condições de laboratório e campo, em solo peneirado e em solo com estrutura indeformada, com a utilização dos métodos TDR-tensiômetro e placa de Richards. Os valores de umidade volumétrica do solo determinados com o método TDR-tensiômetro, foram menores que os obtidos com o método placa de Richards. As leituras de umidade volumétrica do solo franco-arenoso realizadas com o TDR, necessitam de correção.
\end{abstract}

Palavras-chave: placa de Richards, calibração do TDR

\section{Soil water retention curve by TDR-Tensiometer method}

\begin{abstract}
TDR (Time Domain Reflectometry) and tensiometer methods were used to obtain soil water retention curve. Several soil water retention curves of a sandy loam soil were determined in both laboratory and field conditions from sieved and undisturbed soil using a TDR-tensiometer and Richard's pressure plate apparatus. The values of soil water content determined by TDRtensiometer were smaller than those achieved by Richard's pressure plate. The TDR's data of the soil water content for this sandy loam soil require specific correction.
\end{abstract}

Key words: Richard's pressure plate, TDR calibration

\section{INTRODUÇÃO}

A determinação da relação entre umidade volumétrica do solo $(\theta)$ e potencial matricial da água no solo $\left(\psi_{\mathrm{m}}\right)$, mais conhecida como curva de retenção de água no solo, é de grande importância na agricultura irrigada, tanto no que se refere ao dimensionamento de sistemas de irrigação como ao manejo desses sistemas.

O método clássico de determinação desta curva consiste na utilização da placa de Richards para obtenção de vários pontos de equilíbrio entre umidade volumétrica e o potencial matricial da água no solo (Klute, 1986). Este método apresenta algumas desvantagens, como alto custo do equipamento e número reduzido de laboratórios que o possuem, grande tempo necessário para determinação desta curva e problemas de contato hidráulico entre placa porosa e amostra de solo, porém a maior desvantagem seja, possivelmente, a de não representar bem as condições de campo (Cassel, 1985; Bruce e Luxmoore, 1986) razão pela qual várias metodologias, nas condições de laboratório e/ou campo, têm sido propostas (Su \& Brooks, 1980; Bruce \& Luxmoore, 1986; Vieira \& Castro, 1987; Rawls \& Brakensieck, 1989; Martínez et al.,1994; Centurion et al., 1997) na tentativa de tornar a obtenção desta curva mais rápida, simples, barata e/ou precisa.

A utilização do TDR e do tensiômetro na determinação desta curva é promissora, porque pode ser realizada com simplicidade, nas condições de laboratório e de campo; além disso, tais equipamentos podem ser utilizados no monitoramento da umidade e tensão da água no solo e na possível automatização do sistema de irrigação.

A medida de umidade volumétrica com o TDR se baseia na determinação da constante dielétrica do solo $(\varepsilon)$. Os valores da constante dielétrica dos componentes do solo são de 1 para o ar, de 3 a 7 para as partículas minerais e de 80 para a água. Como o componente que possui a maior influência sobre os valores da constante dielétrica do solo é a água, pode-se estimar a umidade volumétrica do solo em função de sua constante dielétrica (Zegelin et al., 1992). 
A equação de calibração da umidade volumétrica em função da constante dielétrica, que a princípio seria válida para os diversos tipos de solo, conforme Topp \& Davis (1985) é a seguinte:

$$
\theta=-5,3 \times 10^{-2}+2,92 \times 10^{-2} \varepsilon-5,5 \times 10^{-4} \varepsilon^{2}+4,3 \times 10^{-6} \varepsilon^{3}
$$

em que:

$\theta \quad$ - umidade volumétrica do solo, $\mathrm{cm}^{3} \mathrm{~cm}^{-3}$

$\varepsilon \quad$ - constante dielétrica do solo.

A utilização do TDR na determinação da umidade volumétrica do solo apresenta muitas vantagens, pois é uma técnica não destrutiva, não utiliza radiação ionizante, apresenta alta exatidão nas medições, é portátil e as medições podem ser feitas de forma automática, em condições de laboratório ou de campo, em qualquer direção no perfil do solo e no tempo real (Gomide, 1998); entretanto, é importante considerar-se que a utilização deste equipamento também apresenta algumas desvantagens, como elevado custo atual do equipamento e possível necessidade de calibração específica para cada tipo de solo, apesar da afirmada universalidade da relação entre umidade volumétrica e constante dielétrica proposta por Topp et al. (1980). Por outro lado, o tensiômetro apresenta a desvantagem da limitação de medidas de potencial matricial entre 0 e $-100 \mathrm{kPa}$.

Em função desses comentários, o presente trabalho de pesquisa teve como objetivo testar a utilização conjunta do TDR e tensiômetro na determinação da curva de retenção da água no solo, fazer comparações entre as curvas obtidas com os métodos TDR-tensiômetro e placa de Richards e determinar a necessidade ou não de calibração específica do TDR para o solo franco-arenoso.

\section{MATERIAL E MÉTODOS}

O trabalho de pesquisa foi desenvolvido no "Centro de Investigación y Desarrollo Agrário de Córdoba", Espanha, com um solo franco-arenoso de origem aluvial, classificado como "Typic Xerofluvent". A análise textural indicou que o solo da camada de $0-20 \mathrm{~cm}$ de profundidade apresenta valores de 16,2, 15,4 e $68,4 \%$ de argila, silte e areia, respectivamente.

A avaliação das medidas de umidade do solo realizadas com o TDR (Trime FM da IMKO - Micromodultechnik) com sondas TDR de 2 hastes e 2 comprimentos de haste (10 e $15 \mathrm{~cm}$ ) foi executada da seguinte maneira: onze vasos de volume conhecido foram enchidos com a mesma quantidade de solo seco em estufa, de forma a proporcionar valor de densidade aparente deste solo, semelhante ao encontrado no campo. Foram adicionadas diferentes quantidades de água em cada vaso, calculadas de forma a proporcionar valores de umidade volumétrica variando entre solo seco e solo saturado. Esses vasos foram cobertos com plástico durante 7 dias, para evitar evaporação e proporcionar a homogeneização da umidade das amostras; posteriormente, realizou-se a medida de umidade do solo, com três repetições, ao se insertar verticalmente a sonda TDR em cada vaso.

O trabalho também consistiu na determinação de várias curvas de retenção de água, em condições de laboratório e campo, em solo com estrutura indeformada e em solo peneirado com malha de $2 \mathrm{~mm}$ de diâmetro, com a utilização dos seguintes procedimentos:

\section{Funil de Haines-placa de Richards}

O funil de Haines e a placa de Richards foram utilizados, respectivamente, para obtenção dos pontos da curva de retenção de água no solo entre 0 e - $10 \mathrm{kPa}$ e entre - 10 e -300 kPa. Utilizaram-se, também, amostras de solo com estrutura indeformada (PR-LI) e amostras de solo peneirado (PR-LP) retiradas na camada de $0-20 \mathrm{~cm}$ de profundidade e contidas em anéis de $4,9 \mathrm{~cm}$ de diâmetro e $2,0 \mathrm{~cm}$ de altura.

As curvas de retenção de água no solo, na fase de secamento, foram determinadas de acordo com o procedimento de equilíbrio seqüencial da umidade e tensão, proposto por Klute (1986).

\section{TDR-tensiômetro em laboratório}

Foram instaladas 2 sondas TDR de 3 hastes metálicas de 20 $\mathrm{cm}$ de comprimento, horizontalmente, na profundidade de 15 $\mathrm{cm}$ e 3 tensiômetros com manômetro de mercúrio, verticalmente, entre as profundidades de 12,5 e 17,5 cm, num solo peneirado e seco à estufa, colocado numa caixa de madeira com 26,5;37,8 e $40,0 \mathrm{~cm}$ de altura, largura e comprimento, respectivamente. Este solo foi saturado no sentido ascendente, mantendo-se o estado de saturação durante $48 \mathrm{~h}$, para ser posteriormente drenado.

Durante o inverno e em condições de laboratório, foram realizadas medidas diárias da umidade volumétrica do solo com o TDR (Trase 6050X1 da Soil Moisture Equipment Corp.) e do potencial matricial da água no solo com os tensiômetros.

O tempo necessário para obtenção das curvas de retenção de água no solo nas fases de secamento (TDR-LP) e de umedecimento foi de, respectivamente, 68 e $45 \mathrm{~d}$.

\section{TDR-tensiômetro em campo (sonda TDR fixa)}

Foram instalados uma sonda TDR de 3 hastes metálicas de $20 \mathrm{~cm}$ de comprimento, horizontalmente, na profundidade de $7,5 \mathrm{~cm}$, e um tensiômetro, verticalmente, entre as profundidades de 5 e $10 \mathrm{~cm}$, no interior de 6 caixas metálicas com 15,20 e $37 \mathrm{~cm}$ de altura, largura e comprimento, respectivamente ( 3 com solo indeformado e 3 com solo peneirado) abertas nas faces inferior e superior .

O solo foi saturado durante $24 \mathrm{~h}$ no sentido descendente e as leituras diárias de umidade e potencial matricial foram realizadas durante a primavera, em condições de campo, respectivamente, com o TDR (Trase 6050X1) e tensiômetros. As curvas de secamento para o solo indeformado (TDR-CI) e peneirado (TDR-CP) foram obtidas em $14 \mathrm{~d}$.

\section{TDR-tensiômetro em campo (sonda TDR móvel)}

Durante o verão e em condições de campo, foram instalados 48 tensiômetros na profundidade de $10 \mathrm{~cm}$, fundamentalmente para fazer o controle do potencial matricial da água no solo, num experimento de alface com irrigação por gotejamento. A cada vez que se desejava realizar uma leitura conjunta da umidade e do potencial matricial, se insertava uma sonda TDR (Trime FM) com 2 hastes metálicas de $10 \mathrm{~cm}$ de comprimento, verticalmente, no solo, com aproximadamente $5 \mathrm{~cm}$ de distância de cada tensiômetro. 
Os valores de umidade volumétrica medidos com o TDR (Trase 6050X1) foram corrigidos de acordo com Gómez et al. (1996) e os valores medidos com o TDR (Trime FM) foram corrigidos com as equações encontradas neste trabalho.

O modelo de estimação da umidade volumétrica em função do potencial matricial, proposto por van Genuchten (1980) foi utilizado para o ajuste das curvas de retenção de água no solo, obtidas pelas distintas metodologias:

$$
\theta=\left\{\left(\theta_{\mathrm{s}}-\theta_{\mathrm{r}}\right) /\left[1+\left(10 \alpha \psi_{\mathrm{m}}\right)^{\mathrm{n}}\right]^{\mathrm{m}}\right\}+\theta_{\mathrm{r}}
$$

em que:

$\theta, \theta_{\mathrm{s}}$ e $\theta_{\mathrm{r}}$ - umidades do solo, do solo saturado e residual do solo, respectivamente, $\mathrm{cm}^{3} \mathrm{~cm}^{-3}$

$\psi_{\mathrm{m}} \quad$ - potencial matricial da água no solo, $\mathrm{kPa}$

$\alpha, \mathrm{n}$ e m - coeficientes da equação com valores de $0<\alpha<1$, $\mathrm{n}>1$ e $0<\mathrm{m}<1$.

\section{RESULTADOS E DISCUSSÃO}

Foram encontrados elevados valores de coeficiente de determinação para a relação entre umidade volumétrica medida com o TDR (Trime FM) e a umidade volumétrica real (Figura 1); isto indica a existência de uma boa correlação entre a umidade volumétrica e a constante dielétrica do solo medida com o TDR, e demonstra boas possibilidades de uso deste equipamento nas medições de umidade do solo, porém as sondas com hastes de 10 e $15 \mathrm{~cm}$ de comprimento superestimaram os valores de umidade volumétrica do solo, em 10 e $38 \%$, respectivamente (Figura 1), mostrando a necessidade da correção específica das leituras realizadas com o TDR (Trime FM) com as hastes metálicas de diferentes comprimentos para o solo franco-arenoso estudado, pois a afirmada equação universal instalada no software deste equipamento não apresentou bons resultados para este solo.

Gómez et al. (1996) também concluíram que o TDR (Trase 6050X1) necessita de calibração específica para este solo

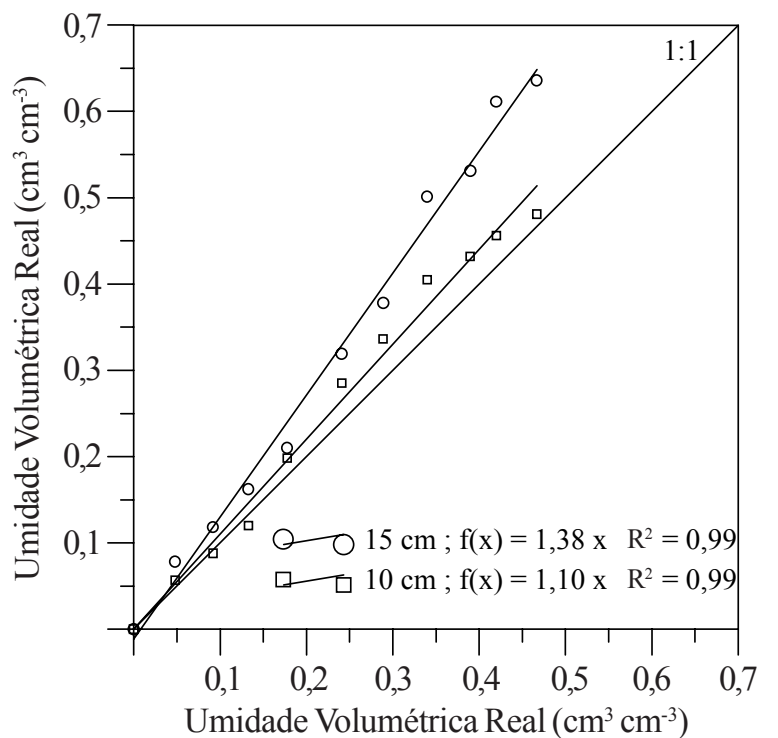

Figura 1. Curvas de calibração do TDR Trime FM para dois comprimentos de haste franco-arenoso, uma vez que o equipamento subestimou os valores de umidade volumétrica do solo; além disso, os resultados encontrados por Silva (1998) para o solo Latossolo Roxo Distrófico, por Fernandes et al. (1998) para um solo argiloso e por Gomide (1998) para o solo Latossolo Vermelho Amarelado, também indicam a necessidade de calibração específica do TDR.

As curvas de retenção de água no solo obtidas com TDR-tensiômetro no laboratório e determinadas por secamento e umedecimento, são apresentadas na Figura 2, na qual se observa que o solo em processo de secamento reteve maior quantidade de água que em processo de umedecimento, fenômeno este conhecido por histerese. O tempo de 68 e 45 d, respectivamente, para determinação dessas curvas, foi grande, devido à pequena evaporação dentro do laboratório, no inverno.

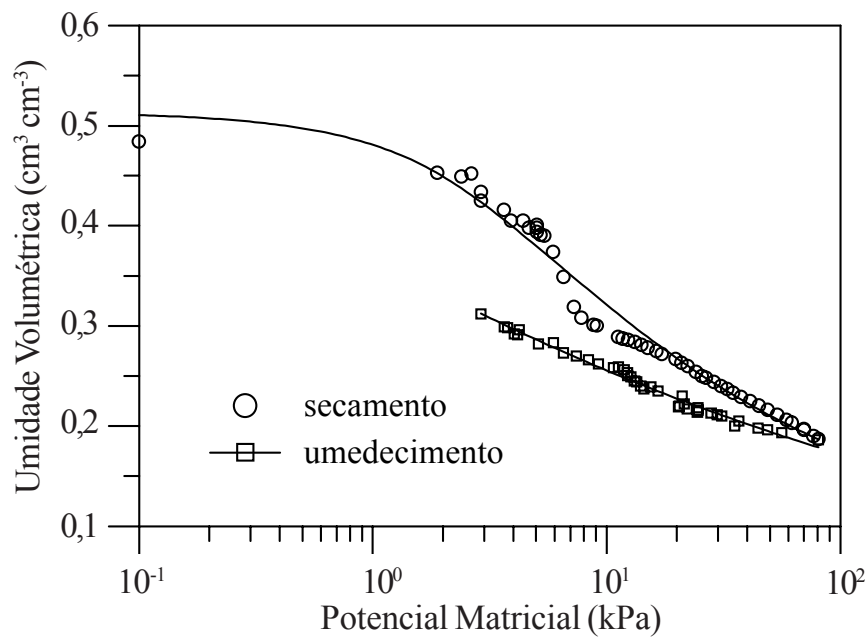

Figura 2. Curvas de retenção de água no solo peneirado, obtidas por secamento e umedecimento, utilizando o método TDR- tensiômetro, em condições de laboratório

A umidade no solo peneirado para um mesmo valor de potencial matricial foi maior nas curvas de secamento obtidas com os métodos placa de Richards, TDR-tensiômetro no laboratório e TDR-tensiômetro no campo (sonda TDR fixa) respectivamente (Figura 3) devido, provavelmente, às diferentes formas de saturação do solo, fazendo com que o solo no campo, que foi saturado no sentido descendente, haja provocado o aprisionamento de ar.

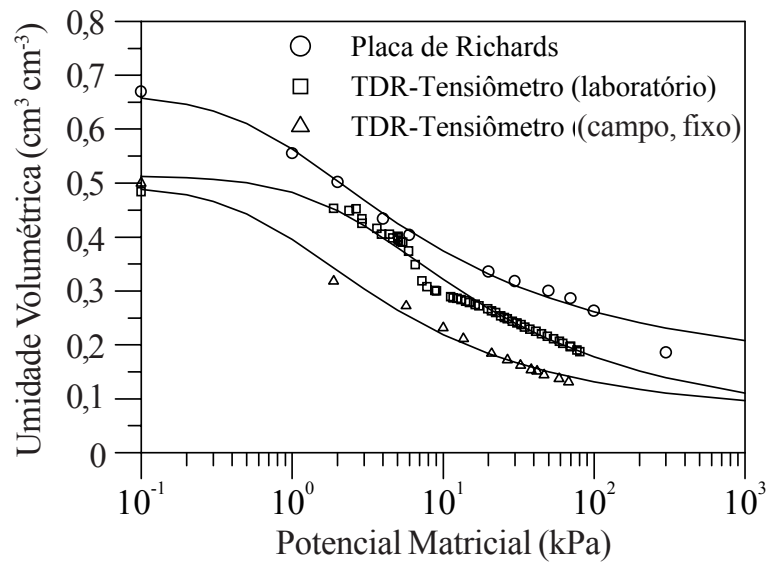

Figura 3. Curvas de retenção de água no solo peneirado, obtidas por secamento, com a utilização de três métodos diferentes 
Da mesma forma, a umidade no solo com estrutura indeformada, para um mesmo valor de potencial matricial, foi maior nas curvas de secamento obtidas pelo método placa de Richards comparadas com as obtidas com o método TDR-tensiômetro no campo (sonda TDR móvel e fixa) (Figura 4); provavelmente, a maior quantidade de ar aprisionado no solo em condições de campo tenha provocado esses resultados. A variabilidade dos pontos de umidade e potencial matricial medidos, respectivamente, com a sonda TDR móvel e os tensiômetros, foi grande, principalmente nas baixas tensões, devido ao constante processo de secamento e umedecimento do solo; à variabilidade do solo superficial e da umidade, no espaço e no tempo; e à própria forma de medição de umidade, pois se utilizava uma sonda móvel.

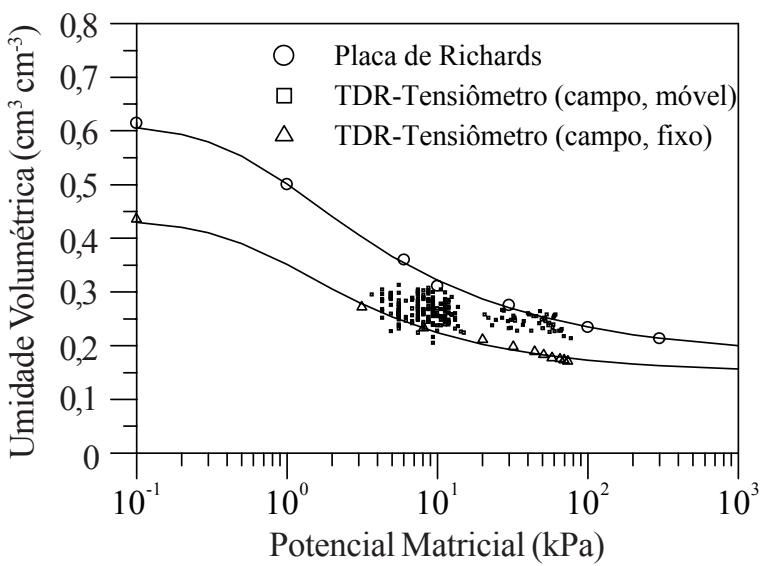

Figura 4. Curvas de retenção de água no solo com estrutura indeformada, obtidas por secamento, com a utilização de três diferentes métodos

Os valores de umidade determinados com a placa de Richards foram maiores que os dados gerais dos solos franco-arenosos e os obtidos com o TDR-tensiômetro (Tabela 1); além disso, os valores dos coeficientes de determinação são altos, indicando a adequação do modelo de van Genuchten no ajuste das curvas de retenção de água no solo.

De forma geral, pode-se constatar que a umidade do solo obtida com a placa de Richards apresenta maiores valores que os obtidos em condições de campo, indicando que, se o controle de irrigação for realizado em função de medidas de umidade do solo e em função dos dados obtidos com o método clássico, poder-se-á cometer o erro de se realizar a irrigação antes do necessário, provocando perdas de água por percolação e encharcamento do solo, razão por que, de acordo com Bruce \&
Luxmoore (1986) é necessário ter-se cautela ao se utilizar dados de laboratório para representar as condições de campo.

\section{CONCLUSÕES}

Os resultados encontrados neste trabalho indicam que:

1. As leituras de umidade volumétrica, realizadas com as sondas do TDR (Trime FM) com hastes de 10 e $15 \mathrm{~cm}$, necessitam de correção específica para o solo franco arenoso estudado.

2. Os valores de umidade volumétrica do solo a um mesmo potencial matricial de água no solo, determinados com o método TDR-tensiômetro, foram menores que os obtidos com o método placa de Richards.

3. O método TDR-tensiômetro pode ser usado para obtenção da curva de retenção de água no solo, em condições de laboratório e de campo.

\section{LITERATURA CITADA}

Bruce, R.R.; Luxmoore, R.J. Water retention: field methods. In: Klute, A. (ed.). Methods of soil analysis. Madison: American Society of Agronomy, 1986. p.563-596.

Cassel, D.K. Physical characteristics of soils of the Southern Region-Summary of in situ unsaturated hydraulic conductivity. North Carolina State University Southern Cooperative Service Bulletin 303, 1985. 143p.

Centurion, J.F.; Moraes, M.H.; Della Libera, C.L.F. Comparação de métodos para determinação da curva de retenção de água em solos. Revista Brasileira de Ciência do Solo, Campinas, v.21, p.173-179, 1997.

Fernandes, A.C.; Oliveira Filho, D.; Pereira, S. Determinação da umidade do solo via reflectometria no domínio do tempo. In: Congresso Brasileiro de Engenharia Agrícola, 27, 1998, Poços de Caldas, MG, Anais ... Resumo expandido. Lavras: UFLA/ SBEA, 1998, v.2, p.326-328.

Gómez, J.A.; Bernardes, M.; Bonachela, S.; Villalobos, F.J.; Mateos, L. Evaluación de três equipos comerciales de TDR para medición de la humedad del suelo. In: Congreso Nacional de Riegos, 14, 1996, Aguadulce, España. Anais... Madrid: Asociación Española de Riego y Drenaje, 1996. p.585-592.

Gomide, R.L. Monitoramento para manejo da irrigação: instrumentação, automação e métodos. In: Congresso Brasileiro de Engenharia Agrícola, 27, 1998, Poços de Caldas, MG, Anais ... Simpósio. Lavras: UFLA/SBEA, 1998, p.133-238.

Tabela 1. Parâmetros da equação de van Genuchten, coeficiente de determinação e valores ajustados de umidade da curvas de retenção de água no solo, obtidas com a utilização da placa de Richards

\begin{tabular}{lcccccccc}
\multirow{2}{*}{ Curvas } & \multirow{2}{*}{$\alpha$} & $\mathrm{n}$ & $\mathrm{M}$ & $\theta_{\mathrm{s}}$ & $\theta_{30}$ & $\theta_{1500}$ & $\theta_{\mathrm{r}}$ & \multirow{2}{*}{$\mathrm{R}^{2}$} \\
\cline { 1 - 4 } & & & & \multicolumn{2}{c}{$\mathrm{cm}^{3} \mathrm{~cm}^{-3}$} & & \\
PR-LP & 0,137 & 1,327 & 0,246 & 0,666 & 0,310 & 0,202 & 0,160 & 0,988 \\
PR-LI & 0,156 & 1,399 & 0,285 & 0,615 & 0,271 & 0,196 & 0,176 & 0,999 \\
TDR-LP & 0,042 & 1,358 & 0,264 & 0,514 & 0,240 & 0,103 & 0,058 & 0,972 \\
TDR-CP & 0,138 & 1,403 & 0,287 & 0,496 & 0,167 & 0,093 & 0,073 & 0,989 \\
TDR-CN & 0,153 & 1,486 & 0,327 & 0,435 & 0,193 & 0,155 & 0,148 & 0,996 \\
F Aren* & - & - & - & 0,453 & 0,207 & 0,095 & 0,041 & - \\
\hline
\end{tabular}

* Rawls \& Brakensiek (1989) 
Klute, A. Water retention: laboratory methods. In: Klute, A. (ed.). Methods of soil analysis. Madison: American Society of Agronomy, 1986. p.563-596.

Martínez, J.; Sanchez, J.M.; Cammeraat, L.H. La medida de la humedad del suelo mediante el método TDR (Time Domain Reflectometry): estado del arte y ejemplos de aplicación. In: Morell, I.(ed.). Investigación en zona no saturada, Publicacions de la Universitat Jaume I, 1994 p.17-27.

Rawls, W.J.; Brakensiek, D.L. Estimation of soil water retention and hydraulic properties. In: Morel-Seytoux, H.J. (ed.). Unsaturated flow in hydrologic modeling. Theory and practice, Kluwer Academic Publishers, 1989. p. 275-300.

Silva, E.L. Determinação automática do teor de água em Latossolo Roxo distrófico com uso de reflectometria de onda. In: Congresso Brasileiro de Engenharia Agrícola, 27, 1998, Poços de Caldas, MG, Anais ... Resumo expandido. Lavras: UFLA/SBEA, 1998, v.1, p.154-156.

$\mathrm{Su}, \mathrm{C}$; ; Brooks, R.H. Water retention measurements for soils. Journal of the Irrigation and Drainage Engineering, New York, v.106, p.105-112, 1980.
Topp, G.C.; Davis, J.L. Time-domain reflectometry (TDR) and its application to irrigation scheduling. In: Hillel, D. (ed.). Advances in irrigation. New York: Academic Press, 1985. p.107-127.

Topp, G.C.; Davis, J.L.; Annan, A.P. Eletromagnetic determination of soil-water content: measurement in coaxial transmission lines. Water Resources Research, Washington, v.16, p.574-582, 1980.

van Genuchten, M.Th. A closed form equation for predicting the hydraulic conductivity of unsaturated soils. Soil Science of Society America Journal, Madison, v.44, p.892-898, 1980.

Vieira, S.R.; Castro, O.M. de. Determinação, em laboratório, de curvas de retenção de água com tensiômetros. Revista Brasileira de Ciência do Solo, Campinas, v.11, p.87-90, 1987.

Zegelin, S.L.; White, I.; Russell, G.F. A critique of the Time-Domain Reflectometry technique for determining field soil water content. In: Topp, G.C.; Reynolds, W.O.; Green, R.E. (eds). Advances in measurements of soil physical properties: Bringing theory into practice. Madison, 1992, p.187-208. 